\title{
23 Dynamik der Sprachnormen in der deutschen Schweiz
}

\begin{abstract}
Diejeweiligen lokalen Standardsprachen Deutsch, Französisch, Italienisch und Rumantsch werden in der obligatorischen Schule gelehrt, damit SchülerInnen „einen Platz in Gesellschaft und Berufsleben finden“ (HarmoS, S-Konkordat, 2007: Art. 3a). Texte in der deutschsprachigen Berufspraxis weichen jedoch von der offiziellen schweizerdeutschen Standardsprache ab. Kann eine Dynamik festgestellt werden, die zu einer sprachpolitischen Diskussion über die schweizerdeutsche Standardsprache führen muss?
\end{abstract}

\section{Die schweizerdeutsche Standardsprache}

Die deutsche Schweiz ist durch eine Diglossie-Situation der Dialekte und des schweizerdeutschen Standards geprägt (vgl. Siebenhaar \& Wyler, 1997; Werlen, 1998; Werlen et al., 2002). Kindern wird bereits in der Grundschule beigebracht, dass der Dialekt als „Mundart“ und der schweizerdeutsche Standard als „Schriftdeutsch“ zu bezeichnen sind, „denn die hochdeutsche Sprache wird auf ihre Funktion in der Schriftlichkeit reduziert“ (vgl. Ziberi-Luginbühl, 1998: 13). Den schweizerdeutschen Standard findet man darum insbesondere in Zeitungen, amtlichen Texten, der Kommunikation von Organisationen, Schulen, Berufsschulen etc. Eine Dynamik in Bezug auf die Diglossie-Situation in der deutschen Schweiz, die sich in den neuen Medien und der Literatur manifestiert, stellt die mediale Diglossie in Frage. Nicht nur in SMS, d.h. in konzeptionell mündlichen, aber schriftlich medialen Situationen, wird vermehrt Dialekt geschrieben. Auch die Rapporte der Aussendienstmitarbeiter (AD-Mitarbeiter - zur Zeit der Datenerhebung sind nur Männer im AD-Team) einer Handelsfirma, die in der Studie „Sprachmanagement im professionellen Netzwerk“ mitgewirkt hat, weisen Muster auf, die nicht dem schweizerdeutschen Standard entsprechen. An welchen Normen orientieren sie sich?

Ein Einfluss auf die Schreibsprache durch die Literalisierung der oralen Dialektkultur wird von Dürscheid (2011: 179) festgestellt. Sie spricht das Thema der Oralität der Dialektkultur und von dialektal verfassten SMS an. Textsorten, die sich zwischen einer konzeptionell mündlichen und einer konzeptionell schriftlichen Ausdrucksweise (vgl. Koch \& Oesterreicher, 1994) befinden, könnten diesem Einfluss besonders ausgesetzt sein. Die Rapporte der AD-Mitarbeiter werden auf diese Vermutung 
hin betrachtet. Untersucht werden soll also einerseits, ob in ursprünglich sowohl konzeptionell als auch medial schriftlichen Situationen, in denen eigentlich die „Schriftsprache“, d.h. der schweizerdeutsche Standard vorherrschen müsste, auch ein vermehrter Einfluss der Mundart festgestellt werden kann. Andererseits geben die Rückmeldungen des Teamleiters einen Hinweis darauf, welche sprachlichen Formen im beruflichen Kontext akzeptiert werden. Die Untersuchungsergebnisse können dahingehend diskutiert werden, ob möglicherweise im Berufsumfeld eine Verschiebung des sprachlichen Normenbewusstseins stattfindet.

\section{Die Untersuchung}

Merkmale der Schriftsprache von Aussendienst-Mitarbeitern einer Firma sollen anhand ihrer Rapporte analysiert und mit den Rückmeldungen des Team-Leiters in einen Bezug gestellt werden. Die in der Studie involvierten Männer (die erste weibliche AD-Mitarbeiterin tritt erst nach der Datensammlung in die Firma ein) sind zwischen 25 und 55 Jahren alt. Der Geschäftsleiter bezeichnet das professionelle und sprachliche Verhalten des Team-Leiters als vorbildlich und sagt, dass er erwarte, dass die AD-Mitarbeiter von ihm lernen sollen. Die Rückmeldungen des Team-Leiters sind damit ein Indikator für den vom Geschäftsleiter gesetzten Massstab, der in Bezug auf die verwendeten Sprachmuster angewendet wird.

Dass der Team-Leiter vom Geschäftsleiter beauftragt wird, die Vorbildrolle einzunehmen, kann mit seiner Ausbildung zusammenhängen. Die Sprachbiografien der beteiligten Personen seien hier darum kurz erwähnt. Der rund fünfzig Jahre alte Team-Leiter absolvierte eine sprachgymnasiale Ausbildung mit Schwerpunkt Latein und Altgriechisch, die durch praxisintensive Ausbildungsgänge auf Fachhochschulebene ergänzt wurde. Seine Kindheit verbrachte er in einem zweisprachigen Teil des Kantons Graubünden, wo hauptsächlich Sursilvan gesprochen wird. Der Team-Leiter war bis 2012 selber AD-Mitarbeiter. Der gleichaltrige Geschäftsleiter ist zweisprachig Italienisch/Deutsch aufgewachsen. Die Team-Mitglieder haben jeweils eine Berufslehre und verschiedene Weiterbildungen durchlaufen. Ein ADMitarbeiter spricht zwei Dialekte. Ein weiterer AD-Mitarbeiter ist zweisprachig (Italienisch/Deutsch) aufgewachsen.

Der Umgang der Geschäftsleitung mit den Rapporten der AD-Mitarbeiter ist inhaltsorientiert, da sie sich für die Resultate der Besuche interessiert, wie die folgende Rückmeldung zeigt: 
RÜ002

Salü [Name]

Deine VIS-Einträge sind in letzter Zeit, zu einem grossen Teil, unpräzise und nichtssagend. Angefügt maile ich dir ein paar Beispiele.

(...)

Wir müssen reden.

Grüsse.

[Signatur AD05]

Der Team-Leiter AD05 äussert sich in seiner Rückmeldung an einen Mitarbeiter ausschliesslich zu den Inhalten seiner Rapporte - sie seien „unpräzise“ und „nichtssagend“. Auf Rückfrage der Studienleiterin antwortet er, dass er v. a. auch die Textqualität der bemängelten Rapporte in Bezug auf Satzbildung, Länge des Textes und Korrektheit mit dem Mitarbeiter mündlich besprechen will, was er mit „wir müssen reden“ in seiner Rückmeldung ankündigt.

Das Zürcher Textanalyseraster erfasst Textqualität über die Grundgrössen des Texts, seine Korrektheit und seine Angemessenheit (Sieber 2008: 273). Die Grundgrössen des Textes sind die folgenden: Rapporte werden von der Firma auf dem hauseigenen Verkaufsinformationssystem VIS, das von allen AD-Mitarbeitern gemeinsam genutzt wird, abgelegt. Es handelt sich also um professionelle, textbasierte Kommunikation in einem „neuen“ Medium. Rapporte können mit Einwilligung des Autors von den anderen Team-Mitgliedern gelesen werden, z.B. wenn Projekte gemeinsam bearbeitet werden. Sowohl der Team-Leiter als auch der Geschäftsleiter haben Zugriff auf alle Rapporte, lesen und kommentieren diese regelmässig. Die AD-Mitarbeiter besuchen pro Tag fünf Kunden. Die gesamte Anzahl der geschriebenen Rapporte für den französisch-, italienisch- und deutschsprachigen Raum ist entsprechend hoch. Pro Jahr werden rund 9000 Rapporte geschrieben. Die Planungszeit, die für das Schreiben der Rapporte eingesetzt wird, ist gering. Als Vorgabe gibt der Team-Leiter den AD-Mitarbeitern jeweils eine Stunde bis eineinhalb Stunden Schreibzeit pro Tag für das Verfassen der fünf Rapporte.

Der Team-Leiter ist regelmässig bei den Besuchen persönlich dabei, kennt die Kunden und den Hintergrund der Projekte, die beschrieben werden. Zudem werden im Verkaufsinformationssystem alle relevanten Daten wie anwesende Personen, Ort des Treffens etc. in einer Maske erfasst. Die AD-Mitarbeiter können darum vom Team-Leiter erwarten, dass dieser anhand der kurzen Texte in den Rapporten

- schnell informiert werden will,

- eigene Ergänzungen anbringt,

- den Text wohlwollend rezipiert und 
- Authentizität und Direktheit höher wertet als sprachformale und ästhetische Stimmigkeit.

Diese Kriterien entsprechen auch den Voraussetzungen, unter denen Parlando häufig vorkommt. Als „Parlando“ wird verschriftete Mündlichkeit bezeichnet (Sieber, 2008: 281). Bei der Analyse der Sprache der AD-Mitarbeiter soll darum berücksichtigt werden, dass die von den AD-Mitarbeitern verwendete Sprachform in Rapporten möglicherweise als verschriftete Mündlichkeit zu bezeichnen ist. Der Team-Leiter beginnt eine seiner Rückmeldungen mit dem dialektalen „salü“: Transfereffekte zum mündlichen Medium des Schweizer Dialekts dürfen damit wohl auch von den AD-Mitarbeitern erwartet werden.

Die Beschreibung der Korrektheit der Sprache von Rapporten der AD-Mitarbeiter muss nicht nur berücksichtigen, dass der schweizerdeutsche Standard sich grundsätzlich bezüglich Wortschatz und Wortbildung, Rechtschreibung und Grammatik von den Standardsprachen in weiteren deutschen Sprachräumen unterscheidet. Das Deutsche ist zwar plurizentrisch (vgl. Scharloth, 2005; Ammon, 1995; 2004), doch die verschiedenen Sprachformen werden über nationale Grenzen hinweg vermischt.

Eine erste Betrachtung der Rapporte ergibt folgende Auffälligkeiten:

- Helvetismen

Beispiele: salü als Begrüssung. starten für beginnen

- Typische syntaktische Konstruktionen wie Verlaufsformen

Beispiele: als [Firmenname] am Verlegen war; die Bekannten in [Ortsname], welche am Laufen sind

- Unachtsamkeit gegenüber der Schreibung von einzelnen Wörtern Beispiele: Zuleitumg; abet; Monzage

- Auslassungen

Beispiele: Keine Projekte geplant; Besuch der Baustelle [Ortsname]; Leitungsführung ist etwas anders als geplant; Technische abklärungen gemacht; Auftrag erhalten; Arbeiten starten; Materialauszug werden wir zu einem späteren Zeitpunkt erstellen; ist noch nicht klar wie weit gebaut wird

- Gross-, / Kleinschreibung

Beispiele: abklärungen, Bestens, Besser, Direkt, Mitgeteilt, Arbeitet, Bezogen, seite

- Interpunktion + typisch dialektale syntaktische Konstruktion

Beispiel: [Name] hat 4 Varianten ausgearbeitet, die möglich sind. Mit korrektem Relativpronomen (üblicherweise wird wo statt die/der/welche/ welcher verwendet). Ein Adjektiv („4 mögliche Varianten“) zu verwenden statt der Konstruktion mit Relativpronomen, wäre einfacher gewesen. Die Versprachlichung mit der Konstruktion legt jedoch die Betonung auf die Möglichkeit.

- Textlänge etc. 
Die Angemessenheit der Textqualität soll in einem nächsten Schritt über die Rückmeldungen des Team-Leiters gemessen werden. Der medial schriftliche Raum des Rapports ist darauf ausgerichtet, dass eine Rückmeldung des TeamLeiters und oft auch der Geschäftsleitung mit hoher Wahrscheinlichkeit erwartet werden darf. Auffälligkeiten sollen in drei Kategorien entsprechend ihrer Nähe zum Sprachusus des Team-Leiters sowie zu den kommentierten, also als negativ sanktionierten Rapporten eingeteilt werden, um festzustellen, wo die Toleranzgrenze liegt.

\section{Schlussfolgerung}

Das „Variantenwörterbuch des Deutschen“ von Ammon et al. (2004) gilt als Referenzwerkfür die Unterscheidung von Wörtern, dieals schweizerdeutscherStandard, als Grenzfälle des Standards oder als mundartlich gelten. Für schweizerdeutsche Besonderheiten im Sprachbereich hat sich der Terminus „Helvetismus“ durchgesetzt. Dürscheid (2011) plädiert dafür, dass es neben dem Variantenwörterbuch auch eine Varietätengrammatik des schweizerdeutschen Standards geben sollte. Wenn eine solche Grammatik des schweizerdeutschen Standards entwickelt würde, dann müsste die Diskussion den aktuellen und authentischen Sprachgebrauch und das aktuelle Normenverständnis in der deutschen Schweiz berücksichtigen. Eine maschinelle Auswertung der im Projekt „Sprachmanagement im professionellen Netzwerk“ erhobenen Sprachdaten könnte zur Entwicklung einer schweizerdeutschen Varietätengrammatik oder einer bedürfnisorientierten Schreibförderung in der beruflichen Bildung beitragen.

\section{Literaturverzeichnis}

Ammon, U., Bickel, H., Ebner, J., Esterhammer, R., Gasser, M., Hofer, L., Kellermeier-Rehbein, B., Löffler, H., Mangott, D., Moser, H., Schläpfer, R., Schloßmacher, M., Schmidlin, R.,Vallaster, G. (2004). Variantenwörterbuch des Deutschen. Die Standardsprache in Österreich, der Schweiz und Deutschland sowie in Liechtenstein, Luxemburg, Ostbelgien und Südtirol. Berlin/New York: de Gruyter.

Ammon, U. (1995). Die deutsche Sprache in Deutschland, Österreich und der Schweiz. Das

Problem der nationalen Varietäten. Berlin/New York: de Gruyter. Decurtins, J. (2017). Sprachmanagement im professionellen Netzwerk. Projektbericht. Dürscheid, C. (2011). Parlando, Mündlichkeit und neue Medien. Schweizerische Zeitschrift für Bildungswissenschaften, 33(2), 175-190.

HarmoS (2007) Interkantonale Vereinbarung über die Harmonisierung der Schule vom 14. Juni. 
Koch, P. \& Oesterreicher, W. (1994). Schriftlichkeit und Sprache. In Günther, H. \& Ludwig, O. (Hrsg.). Schrift und Schriftlichkeit. Writing and its Use. Ein interdisziplinäres Handbuch internationaler Forschung. An Interdisciplinary Handbook of International Research. (S. 587-604) (Bd. 10.1, Handbücher zur Sprach- und Kommunikationswissenschaft). Berlin, New York: de Gruyter.

Scharloth, J. (2005). Zwischen Fremdsprache und nationaler Varietät. Untersuchungen zum Plurizentrizitätsbewusstsein der Deutschschweizer. In Muhr, R. (Hrsg.).

Standardvariationen und Sprachideologien in verschiedenen Sprachkulturen der Welt / Standard Variations and Language Ideologies in Different Language Cultures around the World. Frankfurt am Main u. a.: Lang. S. 21-44.

Siebenhaar, B. \& Wyler, A. (1997). Dialekt und Hochsprache in der deutschsprachigen Schweiz. 5., vollständig überarbeitete Aufl. Zürich: Edition Pro Helvetia.

Sieber, P. (2008). Kriterien der Textbewertung am Beispiel von Parlando. In Janich, N. (Hrsg.). Textlinguistik. Narr: Tübingen.

Werlen, I. (1998). Mediale Diglossie oder asymmetrische Zweisprachigkeit? Mundart und Hochsprache in der deutschen Schweiz. Babylonia 1, 22-35.

Werlen, I., Buri, B., Matter, M. \& Ziberi, J. (2002). Das Projekt Üsserschwyz. Dialektloyalität und Dialektakkommodation von Oberwalliser Migrantinnen und Migranten. (Arbeitspapier 39 der Universität Bern). Bern: Institut für Sprachwissenschaft.

Ziberi-Luginbühl, J. (1998). Zweitsprachunterricht im obligatorischen Schulsystem.

Umsetzungsbericht. Bern: NFP 33. 